\title{
The Predictive Value of Monocyte Count to High-Density Lipoprotein Cholesterol Ratio in Restenosis After Drug-Eluting Stent Implantation
}

This article was published in the following Dove Press journal: International Journal of General Medicine

\author{
Jing $\operatorname{Nan} \mathbb{D}^{1, *}$ \\ Shuai Meng ${ }^{1, *}$ \\ Hongyu $\mathrm{Hu}^{\mathrm{l}, *}$ \\ Ruofei Jia' \\ Ce Chen ${ }^{2}$ \\ Jianjun Peng ${ }^{2}$ \\ Zening Jin' \\ 'Department of Cardiology and \\ Macrovascular Disease, Beijing Tiantan \\ Hospital, Capital Medical University, \\ Beijing, People's Republic of China; \\ ${ }^{2}$ Department of Cardiology, Beijing \\ Shijitan Hospital, Capital Medical \\ University, Beijing, People's Republic of \\ China
}

*These authors contributed equally to this work
Background: The clinical value of monocyte count to high-density lipoprotein cholesterol ratio (MHR) in in-stent restenosis (ISR) of patients who have undergone bare metal stent implantation has been studied. However, the predictive value of MHR in ISR for patients who have undergone drug-eluting stent (DES) implantation has not been explored.

Methods: Non-ST segment elevation acute coronary syndrome (NSTE-ACS) patients who had undergone DES implantation and coronary angiography follow-up at the Beijing Shijitan Hospital, Capital Medical University, between January 1, 2008, and December 31, 2018, were retrospectively enrolled. Patients were divided into ISR and non-ISR groups depending on the follow-up coronary angiography results. Relative clinical information was recorded and analyzed. The receiver operating characteristic curve analysis was used to determine the optimum cut-off pre-procedural MHR to predict ISR.

Results: A total of 214 patients were enrolled in our study. The mean period between two coronary angiography procedures was $25.4 \pm 9.8$ months. Percutaneous coronary intervention due to NSTE myocardial infarction, a bifurcation lesion, increased platelet count, and a high MHR were the independent risk factors in multivariate logistic regression analyses.

Conclusion: Our results indicated that elevated MHR is an independent and useful predictor of ISR in NSTE-ACS patients who have undergone DES implantation.

Keywords: monocyte count to high-density lipoprotein cholesterol ratio, in-stent restenosis, drug-eluting stent

\section{Background}

During the past decades, percutaneous coronary intervention (PCI) emerged as an effective technique to treat a narrowed coronary artery in patients with coronary artery disease (CAD). ${ }^{1}$ The introduction of drug-eluting stents (DES) has remarked reduced the restenosis rate and improved clinical outcomes for patients who had undergone PCI. ${ }^{2}$ However, in-stent restenosis (ISR) remains a major concern in the real-world practice. The incidence rate of ISR is approximately $10 \%$ despite the wide use of the novel technique and medication. ${ }^{3}$

The potential mechanism underlying ISR includes inflammation, extracellular matrix re-modeling, and smooth muscle cell proliferation. ${ }^{4}$ Inflammation is not only a local response in the coronary artery but also is a systemic process. ${ }^{5}$ Monocytes are a major source of the proinflammatory response factors, while high-density lipoprotein cholesterol (HDL-C) exhibits anti-atherosclerotic effects by neutralizing the proinflammatory action of monocytes. ${ }^{6,7}$ Based on these findings, a model using these two
Department of Cardiology and

Macrovascular Disease, Beijing Tiantan Hospital, Capital Medical University, No. II 9 South Fourth Ring West Road,

Fengtai District, Beijing 100070, People's

Republic of China

Tel +86 I5652966904

Email jin_zening@ccmu.edu.cn
International Journal of General Medicine 2020:13 1255-1263 
predictors, the monocyte-to-HDL-C ratio (MHR), has emerged as a novel biomarker in multiple cardiovascular diseases. ${ }^{8-10}$ Lately, the association of MHR and incidence of ISR in patients who have undergone bare metal stent (BMS) implantation has been demonstrated in several studies. ${ }^{11-13}$ However, the predictive value of MHR in the incidence rate of ISR in patients who have undergone DES implantation remains unclear.

The aim of this study is to evaluate the prognostic value of MHR in ISR for non-ST segment elevation acute coronary syndrome (NSTE-ACS) patients who have undergone DES implantation.

\section{Methods}

\section{Study Population}

This was a single-center, retrospective study. Patients who had been diagnosed with NSTE-ACS and subjected to DES implantation as well as coronary angiography follow-up at the Beijing Shijitan Hospital, Capital Medical University, between January 1, 2008, and December 31, 2018, were retrospectively enrolled. Detailed demographic, medical, angiographic, and PCI data were collected from electronic medical records. Hypertension was defined according to the current guideline with systolic blood pressure $\geq 140 \mathrm{mmHg}$ and/or diastolic blood pressure $\geq 90 \mathrm{mmHg}$ for at least two blood pressure measurements or on current anti-hypertensive therapy. Diabetes mellitus was defined as a blood sugar level $\geq 7 \mathrm{mmol} / \mathrm{L}$ in the fasting state or on current anti-diabetic therapy according to the current guidelines. Based on the smoking status, patients were defined as "current smokers" or "ex-smokers" if they had smoked in the past 3 months.

Exclude criteria were as follows: previous PCI, previous coronary artery bypass surgery (CABG), symptomatic heart failure, cardiomyopathy, congenital heart defects, severe valvular heart disease, severe renal (glomerular filtration rate $<30 \mathrm{~mL} / \mathrm{min} / 1.73 \mathrm{~m}^{2}$ ) or hepatic failure, malignancy, hematological disorders, immunological disorder, active infection, no follow-up coronary angiography (CAG), and missing data. Figure 1 shows the study flow chart.

The study protocol was approved by the Ethics Committee of the Beijing Shijitan Hospital, Capital Medical University. The study was implemented in complete concordance with the tenets of Declaration of Helsinki on human research. Informed consent was obtained from the study participants during the hospitalization of CAG follow-up.

\section{Laboratory Test}

All patients who had undergone DES implantation were tested for the whole blood panel, liver and renal functions,

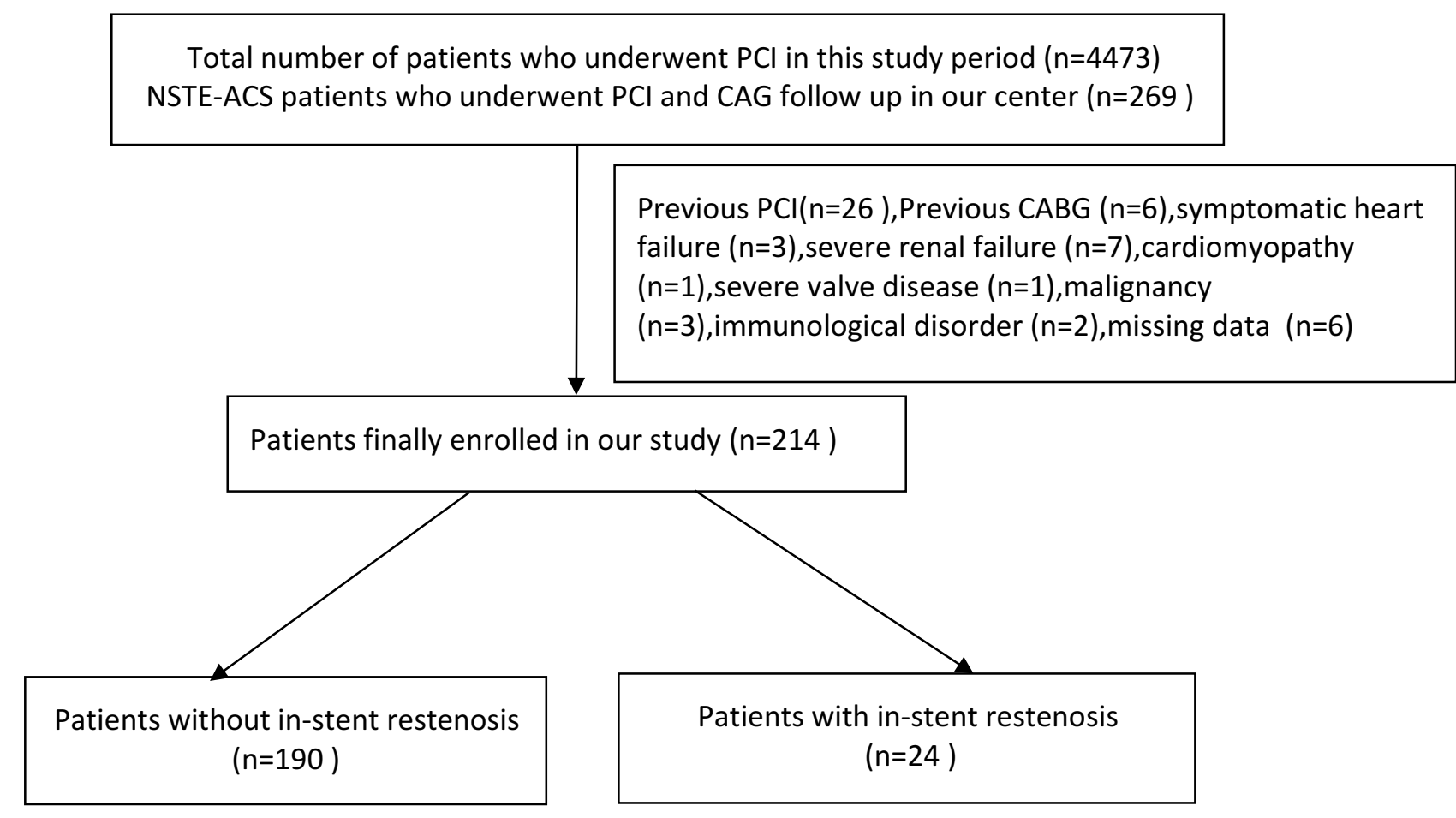

Figure I Study flow chart. 
and glucose, uric acid, and high sensitivity C-reactive protein levels in the fasting state before the procedure as a routine test at our center. All blood samples were collected by trained nurses and measured at the core laboratory of the Beijing Shijitan Hospital according to the manufacturer's instructions. Pre-procedure MHR was calculated by dividing the absolute count of monocytes by the HDL-C value.

\section{Coronary Angiography and $\mathrm{PCl}$ Information}

Two professional interventionists performed coronary angiography according to the standard protocol via the radial or femoral artery. The degree of coronary stenosis was estimated by quantitative coronary angiography (QCA). CAD was defined as the presence of more than $50 \%$ stenosis in the main artery (left anterior descending, left circumflex, or right coronary artery) and/or the main side branch of the main artery. Patients with a vessel narrowed by $70 \%$ or more were subjected to DES implantation. All patients were prescribed with acetylsalicylic acid (loading dose, $600 \mathrm{mg}$; maintenance dose, $100 \mathrm{mg} /$ day) in combination with another $\mathrm{P}_{2} \mathrm{Y}_{12}$ receptor antagonist, such as clopidogrel (loading dose, $600 \mathrm{mg}$; maintenance dose, $75 \mathrm{mg} /$ day) or ticagrelor (loading dose, $180 \mathrm{mg}$; maintenance dose, $90 \mathrm{mg}$ twice/day), before the procedure. PCI was performed in accordance with the current guidelines. Lesion preparation, stent implantation, post-dilatation strategies as well as the use of a glycoprotein IIb/IIIa inhibitor and anticoagulation regimen (heparin, bivalirudin or low-molecular-weight heparin) were left to the operator's discretion. Angiotensin-converting enzyme inhibitors, angiotensin receptor blockers, $\beta$-blockers, statins, and other medications were prescribed based on the guideline recommended. Follow-up CAG was performed only when the patient suffered from recurrent chest pain. The procedure was performed by two experienced interventionists who had been blinded to the results of the baseline test. ISR was defined as restenosis $\geq 50 \%$ inside the stent or $5 \mathrm{~mm}$ proximally or distally in the target vessel according to the result of QCA. The lesion type was diagnosed according to the AHA classification. ${ }^{14}$ The Syntax (SYNergy between PCI with TAXUS and Cardiac Surgery) score was calculated according to the recommendation from previous study. ${ }^{15}$

\section{Statistical Analysis}

Categorical variables are expressed as number and relative frequency (percentage). They were compared using the chi-square test. Data were tested for normal distribution using the Kolmogorov-Smirnov test. Continuous variables are expressed as mean \pm standard deviation or median (quartile 1-quartile 3) based on the normality assumption. They were compared using the independent-samples $t$-test or Mann-Whitney $U$-test, as appropriate. Spearson analysis was used for correlation evaluation between preprocedure MHR and QCA in PCI-vessel and Syntax score in non-PCI vessels. A multivariate Cox regression analysis was performed including parameters that differed significantly between the groups in order to identify the independent predictor for ISR. The receiver operating characteristic curve analysis was used to determine the optimum cut-off MHR in predicting ISR. A $p$-value $<0.05$ (two-sided test) was considered significant. Statistical analyses were performed using SPSS 22.0 (SPSS Inc., Chicago, Illinois).

\section{Results}

A total of 214 patients were enrolled in this study, with an average age of $65.5 \pm 10.3$ years. Table 1 summarizes the baseline demographic, clinical, and angiographic features of the CAD patients who had undergone DES implantation with or without ISR. The rate of NSTE myocardial infarction (NSTEMI) was higher in the ISR group than in the non-ISR group $(50.0 \%$ vs $16.8 \%, p=0.001)$. The rate of presence of a bifurcation lesion was higher and time between the two CAG scans was longer in the ISR group than in the non-ISR group $(37.5 \%$ vs $15.3 \%, p=0.019$; $27.5^{16-31}$ vs $23.0,{ }^{16-25,32-35} p=0.043$, respectively). Angiographic and PCI results, including the rate of calcification lesion, lesion type according to the AHA classification, generation of the implanted stents, the total stent length, maximum stent diameter, and post-dilatation indication, were similar in both groups. We also noticed that the baseline Syntax score in non-PCI vessels was comparable in the two groups however the follow-up Syntax score in non-PCI vessels from ISR group was higher when compared with non-ISR group. The value of follow-up QCA in PCI-vessel is significantly higher in ISR group compared with non-ISR group. Further Pearson correlation analysis revealed that the value of the pre-procedure MHR was associated with the follow-up QCA value in PCIvessel $(r=0.248, p=0.000)$ but not with the difference 
Table I Baseline Demographic, Clinical and Angiographic Features in Coronary Artery Patients Undergoing DES Implantation with and without ISR

\begin{tabular}{|c|c|c|c|}
\hline Variables & Non-ISR Group $(n=190)$ & ISR Group $(n=24)$ & $P$ value \\
\hline Male, n (\%) & $118(62.1)$ & $15(62.5)$ & 1.000 \\
\hline Age (years) & $67.0(58.0-75.0)$ & $63.50(63.00-73.75)$ & 0.976 \\
\hline Hypertension & $144(75.8)$ & $20(83.3)$ & 0.609 \\
\hline Diabetes mellitus & $91(47.9)$ & II (45.8) & 1.000 \\
\hline Smoking & $76(42.1)$ & $14(54.2)$ & 0.123 \\
\hline Left Ventricle Ejection fraction (\%) & $62(55-69)$ & $65(56-68)$ & 0.849 \\
\hline ACEI or ARB, $n(\%)$ & $116(61.6)$ & $17(70.8)$ & 0.383 \\
\hline Beta-blocker & $82(43.2)$ & II (45.8) & 0.830 \\
\hline Statins & $185(97.4)$ & $23(95.8)$ & 0.515 \\
\hline \multicolumn{4}{|l|}{ Target coronary artery, n (\%) } \\
\hline Left anterior descending & $88(46.3)$ & $10(41.7)$ & \\
\hline Left circumflex artery & $39(20.5)$ & $7(29.2)$ & \\
\hline Right coronary artery & $63(33.2)$ & $7(29.2)$ & 0.932 \\
\hline \multicolumn{4}{|l|}{ Reason for stent implantation, $\mathrm{n}(\%)$} \\
\hline Unstable angina pectoris & $158(83.2)$ & $12(50)$ & \\
\hline Non-ST-segment elevation myocardial infarction & $32(16.8)$ & $12(50)$ & 0.001 \\
\hline \multicolumn{4}{|l|}{ Lesion type $(n, \%)$} \\
\hline $\mathrm{A} / \mathrm{B}_{\mathrm{I}}$ & $134(70.5)$ & $16(66.7)$ & \\
\hline $\mathrm{B}_{2} / \mathrm{C}$ & $56(29.5)$ & $8(33.3)$ & 0.813 \\
\hline Calcification lesion (n,\%) & $36(18.9)$ & $9(37.5)$ & 0.058 \\
\hline Bifurcation lesion $(n, \%)$ & $29(15.3)$ & $9(37.5)$ & 0.019 \\
\hline \multicolumn{4}{|l|}{ Number of occluded arteries (n,\%) } \\
\hline 1 & 91 & 10 & \\
\hline 2 & 37 & 8 & \\
\hline 3 & 62 & 6 & 0.985 \\
\hline \multicolumn{4}{|l|}{ Generation of the stents (n,\%) } \\
\hline First generation & $10(5.3)$ & $2(8.3)$ & \\
\hline Second generation & $180(94.7)$ & $22(91.7)$ & 0.629 \\
\hline Total stent length $(\mathrm{mm})$ & $38.0(24.0-57.0)$ & $50.0(33.0-53.8)$ & 0.106 \\
\hline Maximum stent diameter $(\mathrm{mm})$ & $3(2.75-3.5)$ & $3(2.5-3.0)$ & 0.082 \\
\hline Post dilation $(n, \%)$ & $172(92.1)$ & $22(91.7)$ & 1.000 \\
\hline Time between the 2 coronary angiographic studies (months) & $23.0(|8.0-3| .0)$ & $27.5(22.0-37.0)$ & 0.043 \\
\hline Baseline Syntax score in non- $\mathrm{PCl}$ vessels & $8.69(5-11)$ & $9.25(7.25-11.75)$ & 0.400 \\
\hline Follow-up Syntax score in non-PCl vessels & $15.66(12-18)$ & $18.46(14.25-23)$ & 0.012 \\
\hline$\Delta$ Syntax score in non- $\mathrm{PCl}$ vessels & $6.96(4-9)$ & $9.21(6-11.75)$ & 0.004 \\
\hline Follow-up QCA in $\mathrm{PCl}$ vessel & $29.55(21-38)$ & $75.7 \mid(68.75-81.75)$ & 0.000 \\
\hline
\end{tabular}

Note: Bold data displays statistically significant difference $(p<0.05)$.

Abbreviations: ISR, in-stent restenosis; DES, drug-eluting stent; ACEl, angiotensin-converting enzyme inhibitors; ARB, angiotensin receptor blockers; SD, standard deviation; IQR, interquartile range.

between baseline Syntax score and follow-up Syntax score ( $\triangle$ Syntax score) in non-PCI vessels during the follow-up $(\mathrm{r}=0.097, \mathrm{p}=0.159)$.

Table 2 shows comparison results of biochemical and hematological characteristics between NSTE-ACS patients undergoing DES implantation with and without ISR. Most biochemical parameters were similar in the two groups.
The ISR group showed higher monocyte (430.0 [375.0-487.5] vs 390.0 [350.0-460.0], $p=0.047$ ) and platelet (234.5 [197.8-262.0] vs 202.0 [151.0-237.5], $p=0.002$ ) counts compared to the non-ISR group. Other hematological parameters between the two groups were also comparable. The lipid profile, including the total cholesterol, total triglycerides, low-density lipoprotein cholesterol, and 
Table 2 Comparison of Biochemical and Hematological Characteristics in NSTE-ACS Patients Undergoing DES Implantation with and without ISR

\begin{tabular}{|c|c|c|c|}
\hline Variables & Non-ISR Group $(n=190)$ & ISR Group $(n=24)$ & $P$ value \\
\hline Glucose, $\mathrm{mmol} / \mathrm{L}$ & $6.4(5.7-7.7)$ & $6.2(5.3-7.8)$ & 0.827 \\
\hline Creatinine, $\mathrm{mmol} / \mathrm{L}$ & $72.0(56.0-93.5)$ & $81.0(64.3-89.8)$ & 0.726 \\
\hline Total cholesterol, $\mathrm{mmol} / \mathrm{L}$ & $4.9 \pm 0.9$ & $4.9 \pm 1.0$ & 0.993 \\
\hline Triglyceride, $\mathrm{mmol} / \mathrm{L}$ & $1.3(1.0-2.0)$ & $1.3(1.2-2.1)$ & 0.702 \\
\hline Low-density lipoprotein, $\mathrm{mmol} / \mathrm{L}$ & $3.1(2.6-3.6)$ & $3.26(2.4-4.0)$ & 0.596 \\
\hline High-density lipoprotein, $\mathrm{mmol} / \mathrm{L}$ & $1.1 \pm 0.2$ & $1.0 \pm 0.2$ & 0.067 \\
\hline Uric Acid, $\mathrm{mmol} / \mathrm{L}$ & $330.9 \pm 83.7$ & $344.0 \pm 88.4$ & 0.474 \\
\hline Hemoglobin, $g / L$ & $|37.6 \pm| 4.4$ & $144.0 \pm 19.7$ & 0.135 \\
\hline Platelet, $\times 10^{9} / \mathrm{L}$ & $202.0(151.0-237.5)$ & $234.5(197.8-262.0)$ & 0.002 \\
\hline White blood cell, $\times 10^{6} / \mathrm{L}$ & $7890.3 \pm 1715.6$ & $8169.6 \pm 1449.4$ & 0.446 \\
\hline Neutrophil, $\times 10^{6} / \mathrm{L}$, & $5550.0(44 \mid 0.0-8992.5)$ & $5850.0(4575.0-6950.0)$ & 0.442 \\
\hline Lymphocyte, $\times 10^{6} / \mathrm{L}$, & $1620.0(1365.0-1850.0)$ & 1520.0 (|295.0-1897.5) & 0.484 \\
\hline Monocyte, $\times 10^{6} / \mathrm{L}$ & $390.0(350.0-460.0)$ & $430.0(375.0-487.5)$ & 0.047 \\
\hline High sensitivity C-reactive protein, $\mathrm{mg} / \mathrm{L}$ & $5.1(3.7-6.5)$ & $5.5(4.4-7.6)$ & 0.159 \\
\hline Monocyte/HDL-C Ratio & $356.0(287.6-461.7)$ & $424.4(348.0-495.8)$ & 0.019 \\
\hline
\end{tabular}

Note: Bold data displays statistically significant difference $(p<0.05)$.

Abbreviations: NSTE-ACS, non-ST-segment elevation acute coronary syndrome; ISR, in-stent restenosis; DES, drug-eluting stent; SD, standard deviation; IQR, interquartile range.

HDL-C, were comparable between the two groups. MHR was significantly higher in the ISR group than in the nonISR group (424.4 [348.0-495.8] vs 356.0 [287.6-461.7], $p=0.019$ ) (Figure 2).

Univariate logistic regression analyses showed that NSTEMI, bifurcation lesions, platelet counts, and MHRs were significantly associated with the incidence of ISR. Parameters with $\mathrm{p}$ value $<0.05$ in univariable analysis and parameters demonstrated to be associated with the development of ISR in previous studies were included in the multivariable Cox analysis. The result indicated that NSTEMI and higher MHR were the independent risk factors for ISR in the multivariate Cox regression analysis (Table 3).

\section{Comparison of median MHR in two groups}

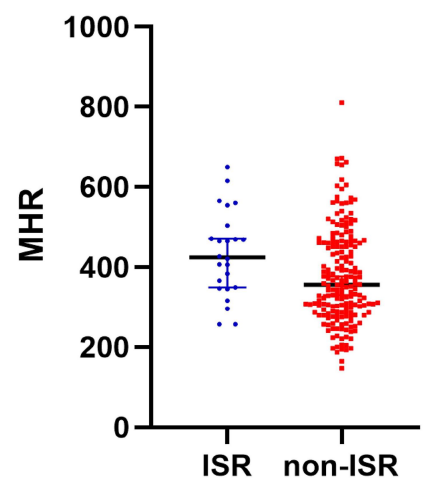

- ISR

- non-ISR

$\mathrm{OR}=1.004$

$95 \% \mathrm{Cl}(1.000-1.007)$ $\mathrm{p}=0.037$

Figure 2 Comparison of the median monocyte count to high-density lipoprotein cholesterol ratio in patients with and without in-stent restenosis.
Receiver operating characteristic curves were used to explore the relation between MHR and ISR. The area under the curve was 0.65 (95\% confidence interval: 0.54-$0.75, p=0.019$ ). Using a cut-off level of 402.5 , the preprocedural MHR predicted ISR with a sensitivity of $62.5 \%$ and specificity of $63.7 \%$ (Figure 3 ).

\section{Discussion}

In this single-centre retrospective study, NSTE-ACS patients who underwent DES implantation and CAG follow-up were divided into ISR and non-ISR group according to the followup CAG result. A total of 214 patients were finally enrolled and 24 of them (11.2\%) developed ISR during follow-up. There are some interesting findings in this study. First, the present study demonstrated that pre-procedural MHR was higher in patients with ISR compared to patients without ISR. Moreover, MHR was an independent predictor of ISR in addition to NSTEMI. The pre-procedure MHR value was associated with the follow-up PCI-vessel QCA value $(\mathrm{r}=0.248, \mathrm{p}=0.000)$. A pre-procedural MHR $>402.5$ predicted ISR with a sensitivity of $62.5 \%$ and specificity of $63.7 \%$. Second, the pre-procedural MHR was not associated with the difference between baseline Syntax score and follow-up Syntax score ( $\triangle$ Syntax score) in non-PCI vessels during the follow-up $(\mathrm{r}=0.097, \mathrm{p}=0.159)$.

Despite the wide usage of new-generation DES and novel medications and techniques, ISR remains an issue for PCI practitioners since it increases the risk of adverse 
Table 3 Univariate and Multivariate Cox Regression Analyses for Predictors of DES Restenosis

\begin{tabular}{|l|l|l|l|l|l|l|}
\hline \multirow{2}{*}{ Variable } & \multicolumn{2}{l|}{ Univariate Analysis } & \multicolumn{2}{l|}{ Multivariate Analysis } \\
\cline { 2 - 7 } & $\mathbf{O R}$ & $\mathbf{9 5 \%} \mathbf{C l}$ & $\mathbf{p}$ value & $\mathbf{O R}$ & $\mathbf{9 5 \%} \mathbf{~ C l}$ & $\mathbf{P}$ value \\
\hline Diabetes mellitus & 1.05 & $0.47-2.34$ & 0.915 & 0.88 & $0.38-2.06$ & 0.771 \\
NSTEMI & 3.22 & $1.41-7.37$ & $\mathbf{0 . 0 0 5}$ & 2.81 & $1.00-7.91$ & $\mathbf{0 . 0 5 0}$ \\
Smoker & 0.77 & $0.33-1.78$ & 0.541 & 1.45 & $0.54-3.88$ & 0.461 \\
Bifurcation lesion & 2.78 & $1.18-6.52$ & $\mathbf{0 . 0 1 9}$ & 1.71 & $0.67-4.36$ & 0.261 \\
Platelet counts & 1.01 & $1.00-1.02$ & $\mathbf{0 . 0 1 6}$ & 1.01 & $1.00-1.01$ & 0.110 \\
MHR & 1.00 & $1.00-1.01$ & $\mathbf{0 . 0 2 1}$ & 1.02 & $1.01-1.03$ & $\mathbf{0 . 0 4 1}$ \\
\hline
\end{tabular}

Note: Bold data displays statistically significant difference $(p<0.05)$.

Abbreviations: DES, drug-eluting stent; NSTEMI, non-ST segment elevation myocardial infarction; CAG, coronary angiography; MHR, monocyte to HDL cholesterol Ratio; $\mathrm{OR}$, odds ratio; $\mathrm{Cl}$, confidence interval.

clinical outcomes, including recurrent angina and even MI. ${ }^{36}$ After stent implantation, mechanically induced vascular damage initiates the local and systemic inflammatory processes, which subsequently result in smooth muscle cell migration and proliferation and finally causes ISR, particularly in patients with persistent systemic inflammation. ${ }^{37}$ Based on the critical role of inflammation in this process, some biomarkers related to inflammatory reactions have been demonstrated to be effective in predicting the incidence rate of ISR. ${ }^{32-35}$

Hematology parameters and their derivatives, including the neutrophil-to-lymphocyte ratio and platelet-tolymphocyte ratio, have been proven to be effective in predicting ISR in several studies. ${ }^{16-20}$ Owing to the inflammatory and thrombotic effects of both monocytes

\section{ROC curve}

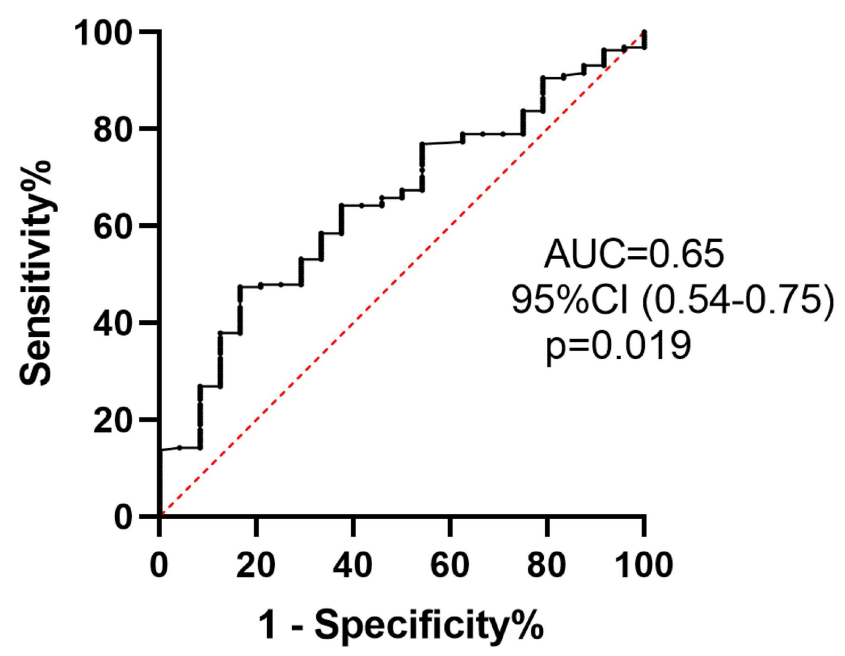

Figure 3 Receiver operating characteristic curve of the median monocyte count to high-density lipoprotein cholesterol ratio in predicting in-stent restenosis in non-ST segment elevation acute coronary syndrome patients who underwent drug-eluting stent implantation. and HDL cholesterol, the novel biomarker MHR has been developed to reflect the local and systemic inflammatory state. As a matter of fact, MHR has been widely investigated in cardiovascular diseases and is found to be related with asymptomatic organ damage in hypertensive patients. ${ }^{21}$ Also, MHR is demonstrated to be useful in predicting coronary artery disease severity and future major adverse cardiovascular events (MACE) in patients with acute coronary syndrome (ACS). ${ }^{22}$ Enhos et al found that MHR was a significant independent predictor of the presence of myocardial bridge (MB). ${ }^{23}$ Saskin et al reported that high preoperative MHR was associated with postoperative atrial fibrillation and mortality in coronary artery bypass grafting. ${ }^{24}$ The relation of MHR with short- and long-term clinical outcome after primary PCI in ST-segment elevation myocardial infarction (STEMI) patients and atrial fibrillation recurrence after ablation procedures is also revealed. ${ }^{25-27}$

All aforementioned studies showed the clinical value of MHR as a novel inflammation-based prognostic marker in cardiovascular diseases. The prognostic value of MHR in predicting ISR in patients who underwent BMS implantation has been evaluated in multiple studies owing to the critical role of inflammation in BMS ISR. ${ }^{11-13}$ However, the predictive value of MHR in restenosis for patients who underwent DES has not been explored. To the best of our knowledge, this is the first study to reveal the relationship between pre-procedure MHR and the incidence of ISR in NSTE-ACS patients who underwent DES implantation.

The underlying mechanism of ISR lesions in DES is slightly different from BMS despite the inflammatory process. Ando et al reported that larger amounts of lipid tissue are present in neointima of a sirolimus-eluting stent (SES) compared to BMS, suggesting that neoatherosclerosis may in part be responsible for ISR after SES implantation. ${ }^{28}$ 
This result was consistent with the finding from another optical computed tomography-based study. ${ }^{29}$ The basic pathological mechanism underlying neoatherosclerosis involves the formation of de-novo lipid-laden macrophages within the neointimal region. ${ }^{30}$ The development of neoatherosclerosis is a risk factor for higher adverse clinical outcomes. ${ }^{31}$ The exact reason for the formation of neoatherosclerosis is unknown. Endothelial dysfunction with poorly formed cell junctions, which results from incomplete endothelial coverage, is believed to be associated with the formation of neoatherosclerosis. ${ }^{31}$ After DES implantation, endothelial dysfunction would cause reduced antithrombotic molecule expression and decreased production of nitric oxide, resulting in the penetration of high levels of lipoprotein and proteoglycans into the subendothelial space, which initializes the development of atherosclerosis. ${ }^{38}$ Increased MHR was associated with endothelial dysfunction. This could partially explain the predictive value of MHR in restenosis after DES implantation. $^{23,24}$ Stent fracture is another important mechanism of ISR in DES implantation which distorts the integrity of the stent structure. The incidence rate of stent fracture is higher in the first generation stents than second-generation stents with $0.7 \%$ to $18.6 \%$ depending on the nature of the studies and the image modality ${ }^{39}$ Intravascular imaging technique is useful in recognization stent fracture in ISR patients. Stent fracture leads to high risk of ISR because it not only decreases the contact of the antiproliferative drug with the arterial wall but also causes the stimulation of the smooth muscle cells which further increases local inflammation process as we mentioned above. ${ }^{5}$ So far as we know, there is no report exploring the association between MHR and stent fracture.

As a systemic inflammatory marker, the pre-procedure MHR should also be associated with the development of atherosclerosis severity in non-PCI vessels. The Spearson correlation analysis revealed that the association was not statistically significant in this study; however, due to the limited number of patients, further studies are still needed to verify this interesting finding.

In conclusion, MHR is a systemic inflammatory marker which can be used in predicting ISR for NSTE-ACS patients who undergo DES implantation.

\section{Limitations}

There are some limitations to this study. First, the sample size was small and STEMI patients were excluded in the study population. Second, it was a single-center study. Third, the definition of ISR was based on QCA assessment rather than more precise quantitative measurements, such as intravascular ultrasound or optical coherence tomography (OCT). Fourth, we measured MHR only at baseline, serial MHR changes were not assessed. Fifth, the impact of MHR on clinical outcomes including death, etc. is more important which needs further exploration. Sixth, CAG follow-up was conducted when ischemic symptoms were suspected, the rate of ISR might be underestimated in clinical practice so a prospective study is needed. Seventh, the value of MHR overlapped between ISR and non-ISR groups. And the AUC of 0.65 indicates that MHR is not high diagnostic performance. This may be associated with the limited number of participants so studies with larger volume of patients is needed to verify these findings. Finally, other inflammatory markers were not evaluated.

\section{Conclusion}

This is the first study to investigate the correlation between pre-procedure MHR and ISR after DES implantation in patients with NSTE-ACS. Our study reveals that elevated MHR had a good clinical value in predicting ISR.

\section{Ethical Approval and Consent to Participate}

The study protocol was approved by the Ethics Committee of the Beijing Shijitan Hospital, Capital Medical University. The study was implemented in complete concordance with the tenets of Declaration of Helsinki on human research. Informed consent was obtained from the study participants during the hospitalization of CAG follow-up.

\section{Acknowledgments}

The information of this study was not presented in any scientific meeting.

\section{Author Contributions}

All authors made substantial contributions to conception and design, acquisition of data, or analysis and interpretation of data; took part in drafting the article or revising it critically for important intellectual content; agreed to submit to the current journal; gave final approval of the version to be published; and agree to be accountable for all aspects of the work. All authors read and approved the final manuscript. 


\section{Funding}

This research was supported by the Chinese Cardiovascular Association-V.G. fund (2017-CCA-VG-042).

\section{Disclosure}

The authors report no conflicts of interest in this work.

\section{References}

1. Bhatt DL. Percutaneous coronary intervention in 2018. JAMA. 2018;319:2127-2128. doi:10.1001/jama.2018.5281

2. Im E, Hong MK. Drug-eluting stents to prevent stent thrombosis and restenosis. Expert Rev Cardiovasc Ther. 2016;14:87-104. doi:10. 1586/14779072.2016.1112267

3. Moussa ID, Mohananey D, Saucedo J, et al. Trends and outcomes of restenosis after coronary stent implantation in the United States. J Am Coll Cardiol. 2020;76(13):1521-1531. PMID: 32972528. doi:10.10 16/j.jacc.2020.08.002

4. Lee MS, Banka G. In-stent restenosis. Interv Cardiol Clin. 2016;5:211-220. doi:10.1016/j.iccl.2015.12.006

5. Shlofmitz E, Iantorno M, Waksman R. Restenosis of drug-eluting stents: a new classification system based on disease mechanism to guide treatment and state-of-the-art review. Circ Cardiovasc Interv. 2019;12(8): e007023. Epub 2019 Jul 26. Erratum in: Circ Cardiovasc Interv. 2019 Oct;12(10):e000044. doi:10.1161/CIRCINTERVENTIONS.118.007023

6. Murray PJ. Immune regulation by monocytes. Semin Immunol. 2018;35:12-18. doi:10.1016/j.smim.2017.12.005

7. März W, Kleber ME, Scharnagl H, et al. HDL cholesterol: reappraisal of its clinical relevance. Clin Res Cardiol. 2017;106:663-675. doi:10.1007/s00392-017-1106-1

8. Ya G, Qiu Z, Tianrong P. Relation of monocyte/high-density lipoprotein cholesterol ratio with coronary artery disease in type 2 diabetes mellitus. Clin Lab. 2018;64:901-906. doi:10.7754/Clin.Lab.2018.171022

9. Sercelik A, Besnili AF. Increased monocyte to high-density lipoprotein cholesterol ratio is associated with TIMI risk score in patients with ST-segment elevation myocardial infarction. Rev Port Cardiol. 2018;37:217-223. doi:10.1016/j.repc.2017.06.021

10. Wu TT, Zheng YY, Chen Y, et al. Monocyte to high-density lipoprotein cholesterol ratio as long-term prognostic marker in patients with coronary artery disease undergoing percutaneous coronary intervention. Lipids Health Dis. 2019;18:180-190. doi:10.1186/s12944-019-1116-2

11. Yilmaz S, Akboga MK, Sen F, et al. Usefulness of the monocyte-to-highdensity lipoprotein cholesterol ratio to predict bare metal stent restenosis. Biomark Med. 2016;10:959-966. doi:10.2217/bmm-2016-0069

12. Ucar FM. A potential marker of bare metal stent restenosis: monocyte count- to-HDL cholesterol ratio. BMC Cardiovasc Disord. 2016;16:186-193. doi:10.1186/s12872-016-0367-3

13. Tok D, Turak O, Yayla Ç, et al. Monocyte to HDL ratio in prediction of BMS restenosis in subjects with stable and unstable angina pectoris. Biomark Med. 2016;10:853-860. doi:10.2217/bmm-2016-0071

14. Ryan TJ, Bauman WB, Kennedy JW, et al. Guidelines for percutaneous transluminal coronary angioplasty. A report of the American Heart Association/American College of Cardiology Task Force on Assessment of Diagnostic and Therapeutic Cardiovascular Procedures (Committee on Percutaneous Transluminal Coronary Angioplasty). Circulation. 1993;88(6):2987-3007. doi:10.1161/01.cir.88.6.2987

15. Morice MC, Serruys PW, Kappetein AP, et al. Outcomes in patients with de novo left main disease treated with either percutaneous coronary intervention using paclitaxel-eluting stents or coronary artery bypass graft treatment in the synergy between percutaneous coronary intervention with TAXUS and Cardiac Surgery (SYNTAX) trial. Circulation. 2010;121(24):2645-2653. doi:10.1161/CIRCULATIONAHA.109.899 211
16. Wang Z, Liu C, Fang H. Blood cell parameters and predicting coronary in-stent restenosis. Angiology. 2019;70:711-718. doi: $10.1177 / 0003319719830495$

17. Qian H, Luo Z, Xiao C, et al. Red cell distribution width in coronary heart disease: prediction of restenosis and its relationship with inflammatory markers and lipids. Postgrad Med J. 2018;94:489-494. doi:10.1136/postgradmedj-2018-135806

18. Murat SN, Yarlioglues M, Celik IE, et al. The relationship between lymphocyte-to-monocyte ratio and bare-metal stent in-stent restenosis in patients with stable coronary artery disease. Clin Appl Thromb Hemost. 2017;23:235-240. doi:10.1177/1076029615627340

19. Li C, Shen Y, Xu R, et al. Evaluation of preprocedural laboratory parameters as predictors of drug-eluting stent restenosis in coronary chronic total occlusion lesions. Angiology. 2019;70:272-278. doi:10.1177/0003319717752245

20. Hu CP, Du Y, Zhu Y, et al. Platelet distribution width on admission predicts in-stent restenosis in patients with coronary artery disease and type 2 diabetes mellitus treated with percutaneous coronary intervention. Chin Med J (Engl). 2018;131:757-763. doi:10.4103/ 0366-6999.228247

21. Aydin E, Ates I, Fettah AM, et al. The ratio of monocyte frequency to HDL cholesterol level as a predictor of asymptomatic organ damage in patients with primary hypertension. Hypertens Res. 2017;40:758-764. doi:10.1038/hr.2017.36

22. Cetin MS, Ozcan Cetin EH, Kalender E, et al. Monocyte to HDL cholesterol ratio predicts coronary artery disease severity and future major cardiovascular adverse events in acute coronary syndrome. Heart Lung Circ. 2016;25:1077-1086. doi:10.1016/j. hlc.2016.02.023

23. Enhos A, Cosansu K, Huyut MA, et al. Assessment of the relationship between monocyte to high-density lipoprotein ratio and myocardial bridge. Arq Bras Cardiol. 2019;112:12-17. doi:10.5935/ abc. 20180253

24. Saskin H, O K S, Yilmaz S. High preoperative monocyte count/ high-density lipoprotein ratio is associated with postoperative atrial fibrillation and mortality in coronary artery bypass grafting. Interact Cardiovasc Thorac Surg. 2017;24:395-401. doi:10.1093/icvts/ ivw376

25. Avci II, Sahin I, Gungor B, et al. Association of monocyte to high-density lipoprotein ratio with bare-metal stent restenosis in STEMI patients treated with primary PCI. North Clin Istanb. 2018;6:393-400. doi:10.14744/nci.2018.93653

26. Çiçek G, Kundi H, Bozbay M, et al. The relationship between admission monocyte HDL-C ratio with short-term and long-term mortality among STEMI patients treated with successful primary PCI. Coron Artery Dis. 2016;27:176-184. doi:10.1097/MCA.00 00000000000343

27. Canpolat U, Aytemir K, Yorgun H, et al. The role of preprocedural monocyte-to high-density lipoprotein ratio in prediction of atrial fibrillation recurrence after cryoballoon-based catheter ablation. Europace. 2015;17:1807-1815. doi:10.1093/europace/euu291

28. Ando H, Amano T, Takashima H, et al. Differences in tissue characterization of restenotic neointima between sirolimus-eluting stent and bare-metal stent: integrated backscatter intravascular ultrasound analysis for in-stent restenosis. Eur Heart J Cardiovasc Imaging. 2013;14:996-1001. doi:10.1093/ehjci/jet003

29. Nakamura D, Dohi T, Ishihara T, et al. Predictors and outcomes of neoatherosclerosis in patients with in-stent restenosis. EuroIntervention. 2020:EIJ-D-20-00539. doi:10.4244/EIJ-D-20-00539

30. Mazin I, Paul G, Asher E. Neoatherosclerosis - from basic concept to clinical implication. Thromb Res. 2019;178:12-16. doi:10.1016/j. thromres.2019.03.016

31. Borovac JA, D’Amario D, Vergallo R, et al. Neoatherosclerosis after drug-eluting stent implantation: a novel clinical and therapeutic challenge. Eur Heart J Cardiovasc Pharmacother. 2019;5:105-116. doi:10.1093/ehjcvp/pvy036 
32. Wang F, Li C, Ding FH, et al. Increased serum TREM-1 level is associated with in-stent restenosis, and activation of TREM-1 promotes inflammation, proliferation and migration in vascular smooth muscle cells. Atherosclerosis. 2017;267:10-18. doi:10.1016/j.atherosclerosis. 2017.10.015

33. Liang S, Aiqun M, Jiwu L, et al. TLR3 and TLR4 as potential clinical biomarkers for in-stent restenosis in drug-eluting stents patients. Immunol Res. 2016;64:424-430. doi:10.1007/s12026-015-8685-6

34. Baktashian M, Saffar Soflaei S, Kosari N, et al. Association of high level of hs-CRP with in-stent restenosis: a case-control study. Cardiovasc Revasc Med. 2019;20:583-587. doi:10.1016/j.carrev.2018.08.015

35. Ornek E, Cetin M, Kiziltunc E, et al. Association of serum procalcitonin level with in-stent restenosis in patients undergoing bare-metal stent implantation. Biomark Med. 2018;12:455-463. doi:10.2217/ bmm-2017-0242
36. Torrado J, Buckley L, Durán A, et al. Restenosis, stent Thrombosis, and bleeding complications: navigating between scylla and charybdis. $\mathrm{J} \mathrm{Am}$ Coll Cardiol. 2018;71:1676-1695. doi:10.1016/j.jacc.2018.02.023

37. Brancati MF, Burzotta F, Trani C, et al. Coronary stents and vascular response to implantation: literature review. Pragmat Obs Res. 2017;8:137-148. eCollection 2017. doi:10.2147/POR.S132439

38. Cui Y, Liu Y, Zhao F, et al. Neoatherosclerosis after drug-eluting stent implantation: roles and mechanisms. Oxid Med Cell Longev. 2016;2016:5924234. doi:10.1155/2016/5924234

39. Omar A, Pendyala LK, Ormiston JA, Waksman R. Review: stent fracture in the drug-eluting stent era. Cardiovasc Revasc Med. 2016;17(6):404-411. doi:10.1016/j.carrev.2016.06.002

\section{Publish your work in this journal}

The International Journal of General Medicine is an international, peer-reviewed open-access journal that focuses on general and internal medicine, pathogenesis, epidemiology, diagnosis, monitoring and treatment protocols. The journal is characterized by the rapid reporting of reviews, original research and clinical studies across all disease areas. The manuscript management system is completely online and includes a very quick and fair peer-review system, which is all easy to use. Visit http://www.dovepress.com/ testimonials.php to read real quotes from published authors.

Submit your manuscript here: https://www.dovepress.com/international-journal-of-general-medicine-journal 